\title{
Research on the Operating Mechanism and Supervision of Platform Economy
}

\author{
Ranyi Wei ${ }^{1, *}$
}

\author{
${ }^{1}$ ESMT Berlin \\ *Corresponding author. Email:www2021abcd@163.com
}

\begin{abstract}
With the continuous development of the Internet, China is paying more and more attention to digital development and the construction of "Internet +". As the Internet and the industry keep integrating with each other, there have appeared various platforms which can connect to more industries. The platforms have gradually developed from a commercial phenomenon to an economic form. This article studies the development process, development characteristics, and operating mechanism of the platform economy, and puts forward some suggestions for regulating asymmetric bargaining and mergers and acquisitions brought by platform economy.
\end{abstract}

Keywords: platform economy, platform economy supervision, platform economy operating mechanism

\section{INTRODUCTION}

In the early days of the information technology revolution, scholars debated the impact of technological progress on economic development. Increasing computing power has made it possible to process and transform large-scale raw data into economic tools. While the algorithm revolution has lowered the threshold for the use of digital tools and traditional tools after data transformation, cloud computing has reduced the cost of computing resources and communication tools and cloud services have achieved economies of scale. It can be said that cloud computing and algorithm revolution are digital economy and that the development of platform economy has provided the foundation and guarantee. Platform economy was initially an e-commerce platform directly oriented to end consumers. However, with the deepening integration of the Internet and industry, the platform industry has become more diversified. As a result, the platform has gradually developed from a commercial phenomenon to an economic form. In other words, the platform developed into the Internet platform and then the platform economy. With the advent of the era of big data, entrepreneurs are pursuing a new "disruptive" business model-platform economy. Platform refers to the digital organization form of different elements, whose core is the digital link of different elements and resources [1]. The platform economy has become the mainstream economic model in the $21^{\text {st }}$ century, which is the most important economy leading the new economic era [2]. For instance, 7 out of the 10 companies with the largest market value are platform companies. Besides, while the average time for creating a traditional company is 129 years, establishing a platform company only takes 22 years [3]. Although the COVID-19 epidemic that broke out at the end of 2019 has had a strong impact on the traditional service industry, it has promoted the development of Internet platform companies. In 2020, the U.S. Nasdaq stock index rose by $2.67 \%$ to 10767 points on July 20. Later, the stock market index rose by 4136 points within three months, an increase of more than $62 \%$, which also exceeded the 9006 points achieved at the end of 2019 and kept setting a record high [4]. It can be seen that in today's society, the platform economy has become the driving force of global economic development

At present, scholars have defined the platform economy from a micro perspective, a macro perspective, an enterprise perspective, an industrial perspective, and an innovation perspective. According to Rochet and Tirole, if the platform charges a higher fee from one side of the market and lowers the price paid by the other side to affect the transaction volume, the market is bilateral. In this definition, the importance of price structure is emphasized; the structure of the platform is based on the premise that both parties participate in it, which means that more than two participants form a platform economy through interest interaction [5]. This definition is based on the bilateral market theory of traditional 
economics, which lacks a description of the essential characteristics of the platform economy. Some scholars believe that the platform economy is based on new infrastructure and driven by technological innovation and business model innovation, which achieves industrial cross-border integration and business innovation by sharing resource on the Internet platform [6]. In other words, the platform economy is an economic platform for resource sharing such as information, assets, and services, which deepens the degree of inter-industry sharing, avoids the information asymmetry and excessive competition in traditional industries, improves the efficiency of asset utilization, and promotes collaborative innovation. With platform companies as the main body and supported by smart technologies such as cloud computing and blockchain, the platform ecosystem can efficiently match scattered demand and supply on a large scale. The sum of all economic activities and economic relations in the Internet economy era is the platform economy. As the scope of resource sharing becomes wider and deeper, the industry boundaries within the industry tend to be more and more blurred and the cross-border integration of the industry through platforms is increasingly significant. Therefore, the platform economy has become an important engine for the development of the new economy. For instance, new models such as e-commerce, online car-hailing, and personalized customization are constantly emerging, which change and reshape the way people behave, the business model of enterprises, and even the nature of many traditional products and services [7].

Against the backdrop of big data, the key to platform economy lies in how to build a platform, attract users, and mobilize users to participate in production. The platform economy builds a liquid market by attracting consumers and manufacturers and connecting them, creates effective channels through digital technology to match transactions between consumers and manufacturers so as to generate transactions and interactions and create value for customers, and develops core algorithm programs and services that support transactions and interactions so as to lower transaction costs, reduce entry barriers, increase the value of platform use, and attract more customers [8]. Therefore, platform economy is a new way to allocate resources with data as a factor of production or a valuable asset on the basis of the rapid development of digital economy and technology.

The platform economy has the characteristics of strong scalability, wide coverage, huge market, low transaction cost, relying on the high participation of users, accurate matching of information between supply and demand, bilateral network externalities, agglomeration radiation effect, and rapid growth. Among them, the most important feature of the platform economy is its dependence on user participation. As a platform for content aggregation and transactions, AppStore has more than 150,000 iOS application developers, more than 650,000 apps and tens of millions of Apple users, thereby enabling both parties to realize transactions through the virtual platform [9]. Google is a global information gathering and sharing platform, which provides advertisers with efficient publishing channels. Therefore, advertising costs is the main source of Google's revenue. In addition, Google provides users with free services such as cloud computing, and optimizes the search engine, thus enhancing customer stickiness. Initially, Alibaba targeted small and medium-sized enterprises, which attracted companies to $\log$ on to the platform with a "free membership system", and provided companies with opportunities to display products. Meanwhile, all kinds of members also brought a continuous flow of information while browsing information. As a result, massive corporate user resources and information flow provide a solid foundation for Alibaba to develop a wider range of business

Precise matching of information is a distinguishing feature of the platform economy. In the market, platform efficiency is embodied as "matching efficiency". The characteristics of the virtual space of the platform economy can break the time and space constraints [10]. The platform is a third-party or fourth-party service that connects upstream and downstream, supply and demand, or buyers and sellers, which is also an operating entity that reduces transaction costs and shares value-added benefits in the process of matching transactions, resource allocation, and open-source innovation. Therefore, companies can break through geographical constraints and increase productivity and efficiency.

The essential feature of the platform economy is the cross-network externalities of the bilateral market. In other words, the increase in the number of users on one side will increase the revenue of users on the other side [11]. For example, the bilateral users of the food delivery platform include consumers and businesses. The externality of consumers to the business is positive, which means that the more consumers there are, the more businesses are willing to enter the food delivery platform. The externality of businesses to consumers is also positive, indicating that the greater the number of businesses, the greater the positive effect it will bring to consumers

The agglomeration radiation effect of the platform economy can be divided into the agglomeration of information resources and the agglomeration of entities [12]. The agglomeration of information resources is realized by building a platform and gathering a large number of scattered information into the platform. The agglomeration of entities means that the upstream and downstream related parties gather to form clusters and realize bundled development through the construction of 
a platform [13]. Platform-based enterprises play a role of connection and integration, which can connect multilateral groups, integrate multi-party resources, and meet the needs of multilateral groups. With the platform economy, companies can reduce intermediate links, create more value, improve efficiency, and achieve added value. Enterprises form a radiation effect by coordinating the upstream and downstream industrial chains, neighboring enterprises, as well as competitors in the same industry.

The platform economy is conducive to creating diversified business models and improving economic efficiency. However, the economic benefits of the platform are non-linear, which possesses network effects and may easily form a market monopoly. The asymmetric bargaining behavior of the platform economy makes it difficult to protect the rights and interests of platform customers. In consequence, there appear problems in privacy protection, network security, property rights protection, consumer rights protection, industry monopoly and other issues. Existing supervision methods can no longer adapt to the governance of the platform economy, so how to promote the healthy and orderly development of the platform economy has become a hot topic in the industry.

\section{THEORETICAL SUPPORT OF PLATFORM ECONOMY}

Platform economics is an emerging discipline born on the basis of bilateral market theory. The bilateral market theory is at the forefront of the industrial organization theory; and the bilateral market model has been enriched with the development of information technology and the Internet. Generally speaking, scholars believe that the bilateral market is a market composed of one or several platforms that promote interactive transactions between end users and that the platform maintains the continuous operation of the bilateral market by charging appropriate fees from both parties' transaction users [14]. However, this definition is only from the perspective of transaction. This article argues that in the bilateral market, the transaction volume of buyers and sellers not only depends on the fees charged by the platform, but also relies on the structure of the market. Rochet and Tirole put forward the principle of non-neutral price structure, which defines the bilateral market from the relationship between price structure and transaction volume. They believe that the transaction volume realized on the platform depends on the total price level, that is, $a=a_{b}+a_{s}$. The total price charged by the platform has a significant impact on the total transaction volume of the platform under the redistribution effect between the buyer and the seller. The total demand and transaction volume of the platform are more sensitive to the redistribution of bilateral user prices. In other words, the demand of users on one side of the bilateral platform will affect the revenue of the other side [15]. For example, a platform company usually set a price that is equal to or lower than its marginal cost for customers in the bilateral market, or even provide platform services to specific groups in the bilateral market for free. In other words, one side of the bilateral market is called the "subsidized party", while the other side is called "spending party".

Armstrong puts forward a definition from the perspective of cross-network externality, who believes that the bilateral market includes two groups of agents connected through a platform and that the number of one group has a great influence on the utility obtained by the other group [16]. This definition is to study the characteristics of different industries in bilateral markets from the perspective of network externalities. Some scholars argue that in the bilateral market the optimal price is not proportional to the marginal cost. Since the network externality is lower than its marginal cost, platform companies have to use pricing power to partially compensate for the common costs of both parties [17]. The indirect network externalities of users in the bilateral market reduce the pricing power of companies on monopolistic platforms. In contrast, competitive platforms tend to impose low prices or take the form of subsidies on the party with less product differentiation and strong network externalities [18]. Some scholars have studied the pricing strategy of food delivery platforms based on the bilateral market theory

Evan divides bilateral markets into three types, including market creation, audience creation, and demand coordination [19]. The market creation type refers to platforms providing services for buyers and sellers with transaction needs, prompting both parties to complete transactions on the platform, attracting bilateral users, using the scale of bilateral users to complete a large number of transactions, and increasing their own interests by charging service fees. The audience creation type refers to platforms attracting more users on one side to meet the needs of users on the other side, whose main goal is to increase the number of users on one side of the platform so as to promote the balanced development of bilateral markets. As for the demand coordination type, platforms provide basic services and the merchants provide products. The two parties constitute a unified entity, which cooperate with each other to meet the needs of consumers [20]. This article takes the market creation type of platforms as the research object the research content is its operation mode. 


\section{RESEARCH ON THE OPERATING MODE AND ELEMENTS OF THE PLATFORM ECONOMY}

Most scholars believe that the operating mechanism of the platform economy is a market price mechanism, that is, the price structure. According to this idea, relative prices will change the relationship between the degree of customer participation in the platform and the fees charged by platform companies to different customers [21]. The price structure is actually an incentive and restraint mechanism, which allocates resources or coordinates interest relationships through network coordination. Taking the online car-hailing platform as an example, the current operating mode of online car-hailing platforms in China can be divided into the $\mathrm{P} 2 \mathrm{P}$ mode and the $\mathrm{B} 2 \mathrm{C}$ mode. $\mathrm{P} 2 \mathrm{P}$ is a franchise model of private cars, meaning that private cars provide transport capacity. The characteristics of this operating model include lower costs and lower prices paid by users. However, it is difficult to control the quality of service. The business model of Didi Express is based on the P2P model and supplemented by the B2C model. In the $\mathrm{P} 2 \mathrm{P}$ mode, platforms obtain a certain percentage of the commission from the order as a service fee; in the B2C mode, platforms share profits with partners such as car rental companies and vehicle manufacturers.

Food delivery platforms also belong to platform economy. As for the initial operating model of such platforms, merchants need to pay a certain amount of entry fee as the entry threshold when entering a food delivery platform. This requirement can increase the conversion cost of a food delivery platform and prevent multiple attribution of merchants. The platform has two trading modes for merchants. One is mainly to provide distribution services for merchants, and to collect distribution fees for profits. The other is to draw commission, where the platform charges merchants a commission in proportion to the transaction volume; in addition, while the distribution costs for small orders are paid by consumers, large orders will be delivered free of charge. In order to increase their core competitiveness, many food delivery platforms have begun to build their own logistics distribution systems, breaking through the distribution bottleneck and obtaining logistics profits through the model of "self-operating + crowdsourcing + agency". As food delivery platforms further develop, more and more merchants log on to these platforms. As a result, food delivery platforms have introduced the model of "bid and ranking". [22] In the early stage, the food delivery platform adopted a sloping pricing strategy of charging merchants and providing free or even subsidies to consumers. In this model, although both consumers and merchants could enjoy the services provided by a platform, the merchants' service fees were the main source of profit for the platform. With the gradual stabilization of the food delivery market, platforms stopped subsidies. In general, food delivery platforms mainly rely on both merchants and consumers to achieve profits. Specifically, they charge consumer transaction fees and charge merchant commissions.

Based on the above case analysis, this article believes that the initial operating model of the platform economy can be shown as follows (see Figure 1), whose effective operation is ensured through the network coordination mechanism set by the algorithm. [23] Specifically, a pre-expected consensus is formed on the digital technology for building a platform, and then the basic customer group is established to reach a key scale. In today's society, the business model of Taobao is that Taobao takes the core leadership and cooperates with Alipay and other functional followers to serve consumers across the country. In other words, it is a model of "single leadership + multiple business services + diversified traders", which is a business model for multilateral mixed market. The difference between the business model of multilateral mixed market and bilateral market lies in that the business model of multilateral mixed market allows relevant interest followers. These followers and platform leaders are complementary to each other in strategy, resources and technology, which makes it easier to build a networked platform economic model and produce network effects [24].

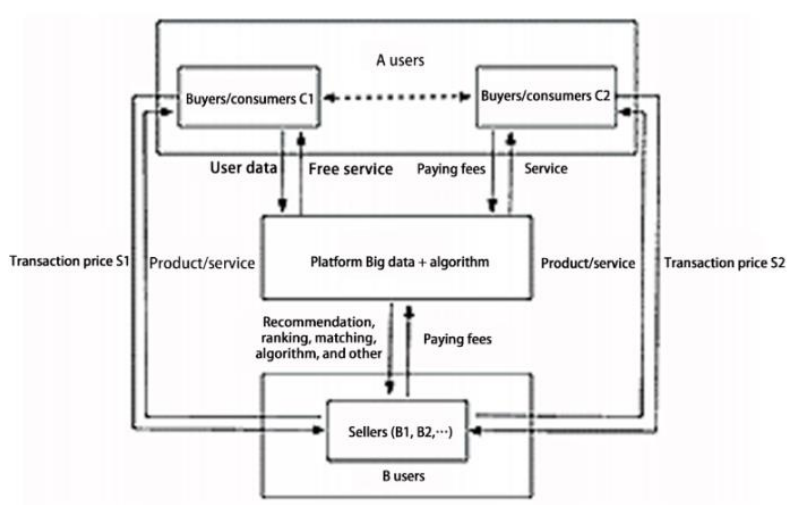

Figure 1 The operating model of the platform econom

To build an operation model of platform economy, it is necessary to seek profit maximization and rapid growth and achieve sustainable development of the environment, society, and economy. Company have created new operating models of platform economy by deploying digital platforms as a means of pursuing value, including circular economy, P2P economy and access economy. These new models are not independent; and a combination of business models may include multiple economic types to achieve a higher degree of diversification of a platform. Many platforms have found new business methods by choosing different combinations. These methods can help maximize the use of existing corporate resources and generate capabilities that are sustainable and can hardly be 
imitated, thereby achieving profit generation and growth [25].

Many scholars have carried out research on the elements of platform economy. This article holds that price, openness, and differentiation are important factors. While the pricing strategy is related to the service fees charged by the bilateral market participants, the price is closely related to the network scale [26]. Open variables are related to the problems of user single-homing and multi-homing. Therefore, platform managers need to solve the trade-off problem between open and closed platforms. These two problems involve the permissions and rules that users should face when accessing, changing and interacting in a platform. An open platform can allow third parties to participate and make contributions, thereby providing possibilities for exploring new market opportunities and expanding the scale of the network. In contrast, a closed platform can help owners use internal resources and protect internal resources from competitors' attacks, thereby preventing it from the infringement of the external value of the network. Since platforms are different from competitors, they need to be differentiated to improve quality or introduce services so as to gain a strategic advantage.

\section{RESEARCH ON THE SUPERVISION OF PLATFORM ECONOMY}

In order to further develop and pursue market share, platforms usually attract attention by using of algorithms. In addition to being a technology, platform algorithms are also value-oriented, which poses great difficulties to supervision. Since asymmetric bargaining behaviors may take place in the platform economy model, the platform, the merchant and the consumer involved in a platform economy model should have a corresponding contract. Therefore, the platform economy has a more complicated legal situation. If a platform gains market share or its customers rely on it, this platform can modify the contract many times. As a result, customers become the vulnerable group that can only passively accept the newly revised terms. Therefore, there is inequality in bargaining between the platform and the customers. At present, with the rapid development of the digital economy, platform economy models represented by Tencent, Alibaba, and Didi have formed an oligopoly or even monopolistic pattern in some fields, and network effects have become the core of the development of platform economic leaders as well as the source of excess profits. As network nodes increase, the value of the network will increase geometrically. As a result, the development pattern of platform economy forces companies to keep expanding, and eventually completely occupy the market to form a monopoly. In fact, the platform economy model has entered more and more traditional industries; and small companies in those industries have either withdrawn from the market or been acquired by a platform. Since 2010, four Internet platforms including Facebook, Amazon, Google, and Apple have made a total of 770 acquisitions; Tencent and Ali have made more than 200 acquisitions in the past decade [27]. All these have aroused concern about the mergers and acquisitions made by Internet platforms. At the same time, there have emerged many problems in the platform economy, including "choosing one of two", "big data-enabled price discrimination against existing customers", algorithmic collusion, inter-company shielding, data leakage, killer acquisitions (killer mergers and acquisitions), etc., which should be dealt with strengthened supervision in accordance with the law. However, in the process of supervision, it is necessary to distinguish the boundaries between business model innovation, normal competitive strategies and illegal and improper operations, and avoid using "moving" or "selective" law enforcement methods for the digital economy.

The core of the platform economy is data. There has always been controversy regarding anti-monopoly research on the platform economy. Can data form a monopoly? Some scholars believe that data monopoly should be regulated. For example, the European Union conducted an investigation into Amazon. However, many people think that it is difficult to determine that data monopoly is the basis of a monopoly since data is a factor of production [29]. This article believes that there is a direct relation between data elements and M\&As made by Internet platforms. Therefore, it is advisable that the characteristics of data elements must be considered during anti-monopoly investigations so as to avoid the risk that the digital economy stops developing due to anti-monopoly. In addition, it is necessary to consider the error cost of anti-monopoly, that is, the cost of wrongly penalizing or restricting normal mergers and acquisitions. Many scholars believe that we should learn from the European Union and the United States antitrust experience. However, this article argues that foreign antitrust experience needs to be viewed dialectically. To deal with antitrust issues, it is worthwhile to take into account China's national conditions and consider the necessity of separately specifying relevant clauses for the platform economy.

\section{CONCLUSION}

In this paper, literature review method is used to sort out the development process of platform economy. Firstly, it is proposed that platform economy has the characteristics of strong ducsibility, wide coverage, huge market, low transaction cost, high participation of users, accurate matching of information between supply and demand, bilateral network externalities, gathering radiation effect, rapid growth and so on. Second, outside sell platform as an example, studied the platform the 
early stages of the economic operation mode, and puts forward the operation mode of the future sustainable development, in the end, this paper argues that the platform economy is a new thing, has more complex legal background, thus for platform of economic management, should be a separate legal provision, shall not copy foreign experience.

\section{REFERENCES}

[1] Tang Fangcheng, Chi Kunpeng. Research on the pricing strategy of online group purchases in a bilateral network environment. Chinese Management Science, 2013, 21(3):185-192

[2] Wu Xiaojun, Fang Yue. Analysis of the pricing strategy of sharing economy platforms based on bilateral market theory. Journal of Nanjing University of Finance and Economics, 2017(05): $28-25$.

[3] Cao Junhao, Chen Hongmin, Shi Peter. Research on the classification and strength of the externality of B2B monopoly platforms based on the bilateral market theory. Journal of Shanghai Jiaotong University, 2010, 44(12): 1661-1664.

[4] Xu Li, Chen Hongmin, Pan Xiaojun. Consumer's multi-party holding behavior and manufacturer's compatibility choice: based on bilateral market theory 2 .

[5] Zhu Linjia. Analysis of factors affecting the price of food delivery platforms based on a bilateral market pricing model. E-commerce, 2015(4): 42-43

[6] Oliver R L. Whence Consumer Loyalty? .Journal of Marketing, 1999, 34(63):33-44.

[7] Poolsombat R, Vernasca G. Partial Multihoming in Two-sided Markets. Discussion Papers in Economics, 2006, 5(10):57-69

[8] Armstrong M. Competition in two-sided markets. The RAND Journal of Economics, 2006, 37(3): 668-691.9.

[9] Wang Na, Tan Liwen. Bilateral Markets: A Conceptual Literature Review. Journal of Lanzhou University of Commerce, 2010, 26(2)26-33.

[10] Huang Minli. Bilateral Market and the Evolution of Market Forms. Journal of Capital University of Economics and Business, 2007, 9(3):43-49.

[11] Dong Liang, Zhao Jian. Bilateral market theory: a review. World Economic Literature Collection. 2012 (01): 53-61

[12] Fu Yu, Sui Guangjun, Zhao Zile. Single-oligopoly Competitive Monopoly: The Theoretical Construction of New Market Structure-Based on the Investigation of Internet Platform Enterprises. China Industrial Economy.2014(1):140-152

[13] Bolt W, Tieman A F. Heavily skewed pricing in two-sided markets. International Journal of Industrial organization, 2008(05): 1250-1255.

[14] Roson R. Two-sided markets: A tentative survey.Journal of Network Economics, 2005(04): $142-160$

[15] Chi Kunpeng. Research on online group buying pricing strategy based on bilateral market theory. Beijing Jiaotong University, 2012.

[16] Hongling Chen, Xiangjian Zhang, Xiao Liu. Overview and Future Prospects of Frontier Research on Platform Economy. Journal of Yunnan University of Finance and Economics, 2019(5): $3-11$

[17] Meng Fanxin. An example of Taobao platform for online trading market governance under the sharing economy model. Reform, 2015, (12): 104-111.

[18] Dong Weigang, Xu Yuhai, Sun Jia. Research on Bilateral Pricing Mechanism under Inter-industry Platform Cooperation_—Based on the Analysis of the Impact on Inherent Income. China Industrial Economy, 2011, (7): 65-75.

[19] Hongling Chen, Xiangjian Zhang, Xiao Liu. Overview and Future Prospects of Frontier Research on Platform Economy. Journal of Yunnan University of Finance and Economics, 2019, (5): 3-11.

[20] Fu Yu, Sui Guangjun, Zhao Zile. Single-oligopoly Competitive Monopoly: The Theoretical Construction of New Market Structure_-Based on the Investigation of Internet Platform Enterprises. China Industrial Economy.2014(01):140-152. · .

[21] Ye Xiumin. Analysis of the characteristics of platform economy. Journal of Hebei Normal University: Philosophy and Social Sciences Edition, 2016, (2): 114-120.

[22] Li Yunyao, Liu Haihai, Huang Shaojian. Research trends in platform economic theory. Economics Trends, 2013, (7): 123-129.

[23] Wei Xiaoyu. Cooperative governance in the Internet platform economy. Administration and Law, 2018, (10): 43-50

[24] Li Wen, Xing Xiaohan, Mei Lei, etc. Research on Innovation Path of Retail Platform Enterprise Business Model under the Background of Network Economy, Business Economic Research, 2018 (16): 105 107. 
[25] J. Tirole, J. Rochet. Two-sided markets: A progress report. Post-print, HAL, 2006.

[26] Rochet, J. C., Tirole, J. Platform competition in two-sided markets. Journal of the EuropeanEconomic Association, 2003: 990 - 1029.

[27] Wu Xiaojun, Fang Yue. Analysis of the pricing strategy of sharing economy platforms based on bilateral market theory. Journal of Nanjing University of Finance and Economics, 2017(05): 28-25. 